DOI: $10.2478 / \mathrm{v} 10282-012-0025-\mathrm{y}$

\title{
Surgical management of tuberculum sellae and planum sphenoidale meningiomas
}

\author{
Adrian Bălașa, Rareș Chinezu' ${ }^{1}$, Dorin Nicolae Gherasim² \\ Neurosurgery Departement, Targu Mures Clinical Emergency Hospital \\ ${ }^{1} 6^{\text {th }}$ year neurosurgery resident \\ ${ }^{2} 2^{\text {nd }}$ year neurosurgery resident
}

\begin{abstract}
Introduction: Tuberculum sellae and planum sfenoidale meningiomas represent $5 \%$ to $10 \%$ of intracranial meningiomas and represent a subgroup of anterior skull base meningiomas. Approximately two thirds of patients complain of failing vision in one eye as the first symptom, and monocular blindness may be present in half of patients before surgery. Due to the constant antomical relationship of these tumors with the optic nerves there is a classic presentation of these tumors represented by the chiasmal syndrome.

Material and methods: In this study, we have retrospectively analyzed 18 cases consecutively operated between 2006 and 2012 at the Targu Mures Neurosurgery Department. Considering the length of the visual disturbances we have divided the study group in two categories: early decompression with visual distubances expressed within at most 12 months prior to surgery, and late decompression with visual disturbances of more than 1 year.

Surgical technique: Sugical approach has been performed on the side with the most visual deficit, and if visual deterioration was found to be approximative on both sides we chose the right side for surgical approach. In all the patients, we have performed a fronto-pterional craniotomy. In all the
\end{abstract}

cases we chose a subfrontal approach. Tumor has been further resected using standard microsurgical techniques, total tumoral resection being the preoperative goal in all surgical interventions

Results: Out of the 18 cases operated, 13 cases were of tuberculum sellae meningiomas, while 5 cases were of planum sphenoidale meningiomas.

All patients in the early decompression group (9 cases) have presented visual improvement, whilst of the late decompression group 5 cases (55,5\%) presented constant visual deficit, 2 cases $(22,25 \%)$ presented visual improvement and 2 cases $(22,25 \%)$ presented a decrease of the visual deficit.

The visual disturbances have improved in 11 cases $(61.1 \%)$, in 5 cases $(27,7 \%)$ the visual deficit has remained constant, and in 2 cases $(11,2 \%)$ the visual deficit has worsened postoperatively. Conclusions: We consider that the optimal approach should be based on tumor anatomy and surgeon experience and from this point of view frontolateral and pterional approaches provide remarkable improvement compared to the bifrontal approach. Regardless of the selected surgical approach it is essential to avoid injury to the blood supply of the optic apparatus.Endoscopy will have a role in skull base surgery with 
proper indications and well trained surgeons.

Key words: tuberculum sellae, planum sphenoidale, chiasmatic syndrome, subfrontal approach

\section{Introduction}

Tuberculum sellae and planum sphenoidale meningiomas represent $5 \%$ to $10 \%$ of intracranial meningiomas and represent a subgroup of anterior skull base meningiomas $(10,11,19)$.

Tuberculum sellae meningiomas are located not only on the limited surface between the prechiasmatic sulcus and and diaphragm sellae, but also on the limbus sphenoidalis, chiasmatic sulcus and diaphragma sellae. Planum sphenoidale meningiomas are located more anterior and in proximity of the olfactory groove location $(4,10)$.

The mean age at diagnosis is in the fourth decade, and women/men ratio affected by this patology is $3: 1$. Due to the constant antomical relationship of these tumors with the optic nerves there is a classic presentation of these tumors represented by the chiasmal syndrome, a primary optic atrophy with bitemporal field defects in adult patients with a radiologically normal sellae (18).

In most patients visual loss has an insidious onset, and the course is progressive. It may, however, be acute or fluctuating. Approximately two thirds of patients complain of failing vision in one eye as the first symptom, and monocular blindness may be present in half of the patients before surgery.

Preoperative signs of pituitary involvement and failure are rare $(12,17)$.

Because the visual loss is usually slowly progressive, some tumors may reach a large volume before diagnosis and treatment.

In this study we present our surgical experience in the treatment of 18 patients with tuberculum sella and planum sfenoidale meningiomas using a subfrontal aproach. We have evaluated the visual deficit pre and postoperatively as well as the degree of resection.

\section{Material and methods \\ Clinical data}

In this study, we have retrospectively analyzed 18 cases consecutively operated between 2006 and 2012 at the Targu Mures Neurosurgery Department. We have excluded from our study 3 cases initially operated elsewhere and recidivated cases operated in this period.

All the patients in the study group have been imagisticaly examined preoperatively with CT and MRI studies. As described in the literature, we considered tubercullum sellae meningiomas the ones located on the small surface between the chiasmatic sulcus and diaphragma sellae. We classified as planum sphenoidale meningiomas, the ones localised more anteriorly near to the olfactory groove location.

We have determined the sizes of the meningiomas by analyzing the preoperative MRI and calculating the largest tumoral diameter found.

At one patient we have performed a digital substraction cerebral angiography to determine the patency of one of the internal carotid arteries. No kind of embolization has been attempted at any of the cases.

The symptomatology of the patients at admission is presented in Table 1, radiological findings being presented in Table 2. Severe visual deficit was considered the inability to count raised fingers at an arm length distance. 
TABLE 1

Clinical findings

\begin{tabular}{|c|c|}
\hline \multicolumn{2}{|l|}{ Duration of visual deficit } \\
\hline$<3$ months & 1 \\
\hline 3 months -12 months & 8 \\
\hline $2-48$ months & 6 \\
\hline$>4$ years & 3 \\
\hline \multicolumn{2}{|l|}{ Type of visual deficit } \\
\hline Unilateral partial visual deficit & 2 \\
\hline Bilateral partial visual deficit & 7 \\
\hline Unilateral severe visual deficit & 2 \\
\hline $\begin{array}{l}\text { Severe visual deficit on one side with partial } \\
\text { visual deficit on the contralateral side }\end{array}$ & 3 \\
\hline Bilateral severe visual deficit & 4 \\
\hline \multicolumn{2}{|l|}{ Other clinical symptoms } \\
\hline Personality changes & 5 \\
\hline Epileptic seizures & 4 \\
\hline Motor deficit & 1 \\
\hline \multicolumn{2}{|l|}{ Other important comorbidities } \\
\hline Hypertension & 3 \\
\hline Obesity & 6 \\
\hline Type 2 diabetes & 2 \\
\hline
\end{tabular}

TABLE 2

Radiological findings

\begin{tabular}{|l|c|}
\hline Localization & \\
\hline Tuberculum sellae & 13 \\
\hline Planum sphenoidale & 5 \\
\hline Internal carotid encasement & 1 \\
\hline Involvement of the optic canal & 4 \\
\hline Hydrocephalus (moderate) & 2 \\
\hline
\end{tabular}

Considering the length of the visual disturbances we have divided the study group in two categories: early decompression with visual disturbances expressed within at most 12 months prior to surgery, and late decompression with visual disturbances of more than 1 year.

\section{Surgical technique}

All patients have been operated using general gaseous anesthesia (Sevoflurane). Patients have been positioned in supine position the head being fixed in a three-pin head holder, flexed to the side and gently extended downwards in order to maximize the view of the orbital roof and reduce the amount of traction.

Sugical approach has been performed on the side with the most visual deficit, and if visual deterioration was found to be approximative on both sides we chose the right side for surgical approach.

In all the patients, we performed a fronto-pterional craniotomy. Using preoperative CT examinations, we tried not to open the frontal aeric sinus, but this was not always possible. When opened, the frontal aeric sinus was plugged with gelatin sponge soaked in antibiotic (gentamicin) and homologous fatty tissue.

The dura mater was incised in curvilinear fashion, with an inferior oriented pedicle. The basal cisterns were opened and CSF suctioned. In order to further enhance the surgical field and to reduce the traction applied to the brain, the Sylvian fissure was opened in all the cases.

In all the cases we chose a subfrontal approach, the frontal lobe being gently tractioned with the use of an autostatic retractor, whilst attempting to preserve the olfactive nerve.

With the tumor expressed we proposed that prior to the start of the tumoral resection an inspection and a blunt disection of the tumoral margins be performed so that as many as possible of the key anatomical landmarks could be identified as early as possible during surgery (Figure 1).

Tumoral resection was started at the level of the tumoral insertion on the dura mater, further dissection space being made via debulking of the tumor (Figure 2).

Dissection of the tumor from the optic nerves was performed using a combination of blunt and sharp dissection techniques. The optic nerve was not resected in order to enhance the surgical 
field even in the 2 cases with complete visual loss. Optic nerve unroofing was performed in the cases with tumoral invasion of the optic canal. The tumor has been further resected using standard microsurgical techniques, total tumoral resection being the preoperative goal in all surgical interventions (Figure 3).

After the resection of the meningioma the dural insertion was further coagulated in order to reduce the tumoral recurrence rate. Dura mater was sutured in a watertight fashion and the bone flap was repositioned in all the cases.

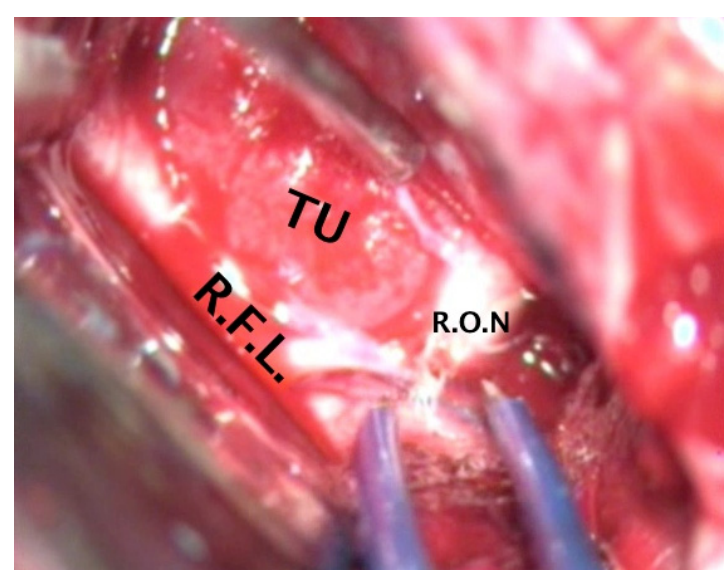

Figure 1. Tumoral expression R.O.N right optic nerve, R.F.L right frontal lobe, TU tumor

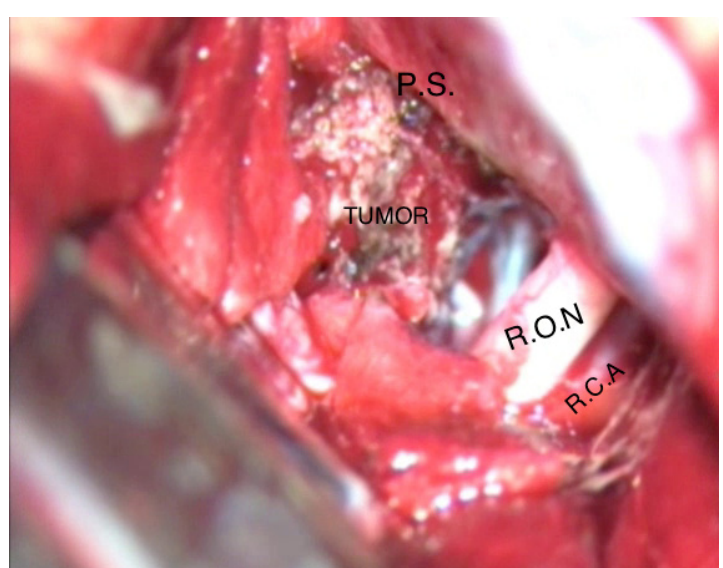

Figure 2. Partial tumor resection and decompression of right optic nerve R.O.N right optic nerve, R.F.L right frontal lobe, TU tumor, P.S. planum sphenoidale

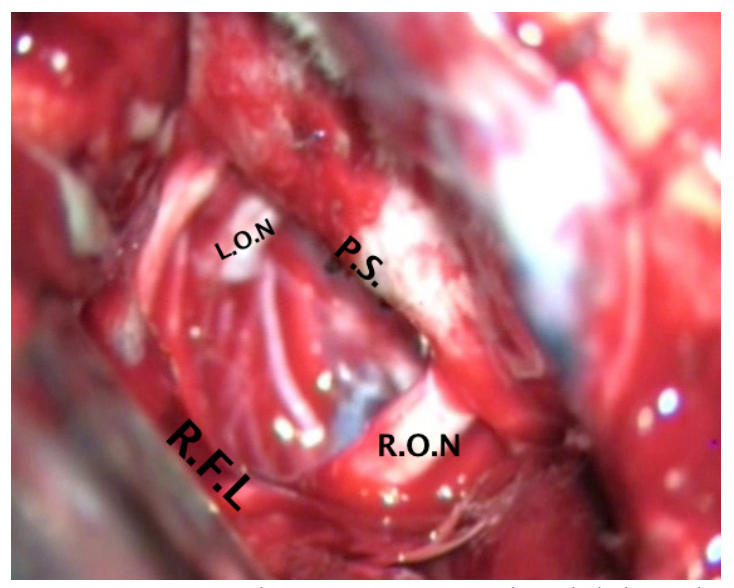

Figure 3. Complete tumor removal with bilateral optic nerves decomprssion R.O.N right optic nerve, L.O.N left optic nerve ,R.F.L right frontal lobe, TU tumor, P.S. planum sphenoidale

\section{Results}

In this study we have included 18 patients with ages between 31 and 64 years old. We have observed greater prevalence in women - 13 cases versus 5 cases in men. Summarized results are found in Table 3.

Out of the 18 cases operated, 13 cases were of tuberculum sellae meningiomas, while 5 cases were of planum sphenoidale meningiomas.

The average tumor size determined on the preoperative IRM scans was determined in our series to be of $2.4 \mathrm{~cm}$.

The visual disturbances improved in 11 cases $(61.1 \%)$, in 5 cases $(27,7 \%)$ the visual deficit remained constant, and in 2 cases $(11,2 \%)$ the visual deficit worsened postoperatively.

All patients in the early decompression group (9 cases) presented visual improvement, whilst of the late decompression group 5 cases $(55,5 \%)$ presented constant visual deficit, 2 cases $(22,25 \%)$ presented visual improvement and 2 cases $(22,25 \%)$ presented a decrease of the visual deficit.

In 14 cases $(77,7 \%)$ we achieved complete resection documented by 
postoperative CT scan. In 4 cases $(22,3 \%)$ subtotal resection was performed

No significant intraoperative events were found in the operation logs. One patient severely deteriorated 3 days postoperatively due to a thrombosis of the cavernous segment of the internal carotid artery. An emergency decompressive craniectomy was performed but the patient deceased after 7 days from the initial operation. Another patient presented a scalp infection that required secondary suture at 9 days since the initial surgical intervention.

All the remaining patients have been followed-up for a period ranging between 1 year to 6 years, neurosurgical and imagistic reevaluations being performed at 3 months, 6 months and yearly on the respective patients.

One patient developed a tumor regrowth at 3,5 years from the initial surgical intervention, the second surgery not being included in the study.

In figures 4,5 and 6 we present an illustrative case of gross total removal of a planum sphenoidale meningioma.

TABLE 3

Results

\begin{tabular}{|c|c|c|}
\hline \multicolumn{2}{|c|}{ Visual deficit whole series } & \\
\hline \multicolumn{2}{|c|}{ Improved } & 11 cases $(61,1 \%)$ \\
\hline \multicolumn{2}{|c|}{ Constant } & 5 cases $(27,7 \%)$ \\
\hline \multicolumn{2}{|c|}{ Worsened } & 2 cases $(11,2 \%)$ \\
\hline $\begin{array}{l}\text { Visual } \\
\text { deficit }\end{array}$ & \begin{tabular}{|l|} 
Early \\
decompression
\end{tabular} & $\begin{array}{c}\text { Late } \\
\text { decompression }\end{array}$ \\
\hline Improved & 9 cases $(100 \%)$ & 2 cases $(22,25 \%)$ \\
\hline Constant & - & 5 cases $(55,5 \%)$ \\
\hline Worsened & - & 2 cases $(22,25 \%)$ \\
\hline \multicolumn{3}{|c|}{ Tumor removal } \\
\hline & Total & $14(77,7 \%)$ \\
\hline & ubtotal & $4(22,3 \%)$ \\
\hline
\end{tabular}
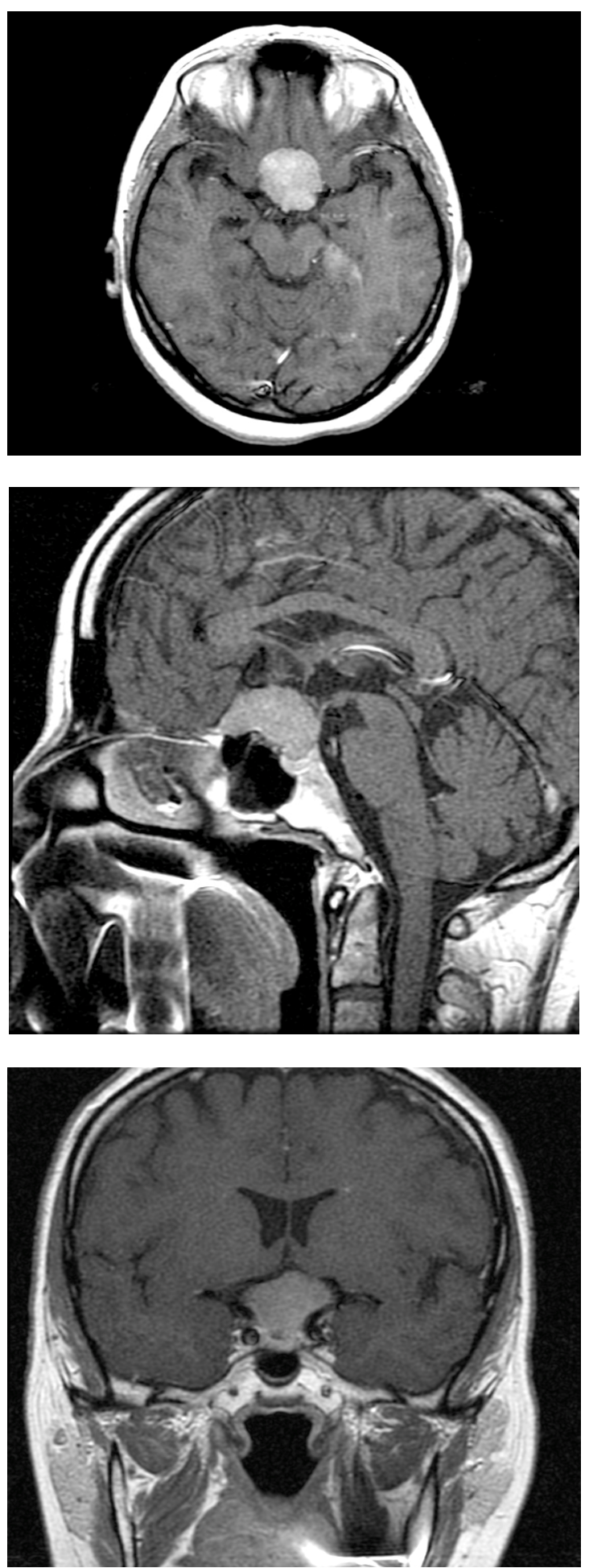

Figure 4. Illustrative case of a planum sphenoidale meningioma with bilateral visual disturbances and severe left visual deficit 


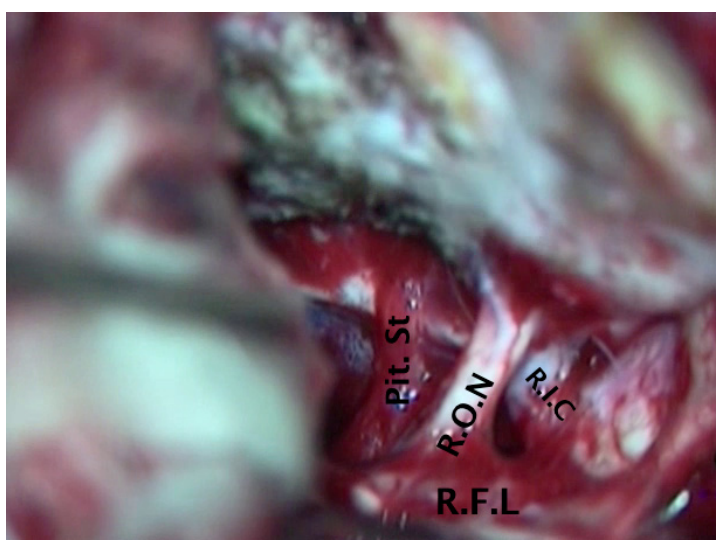

Figure 5. Complete tumor removal with preservation of the pituitary stalk. R.O.N - right ontic nerve, Pit St -pituitary stalk, R.I.C - right internal carotid, R.F.L right frontal lobe
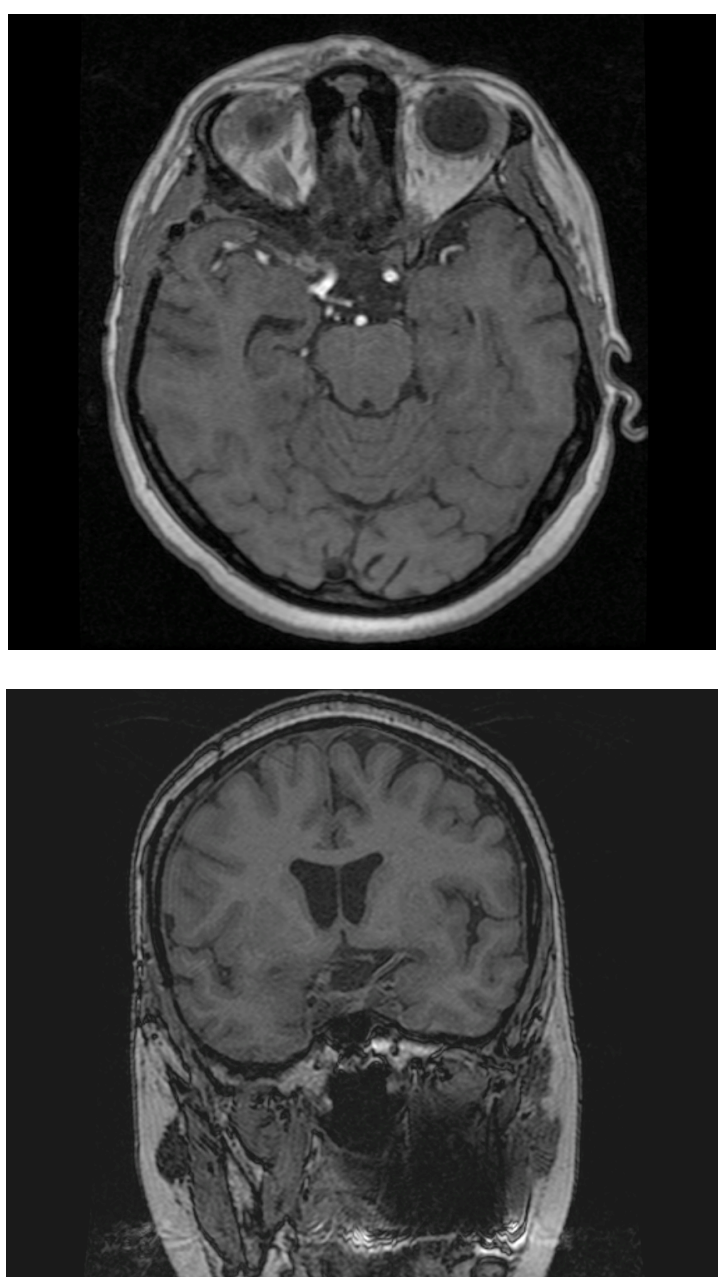

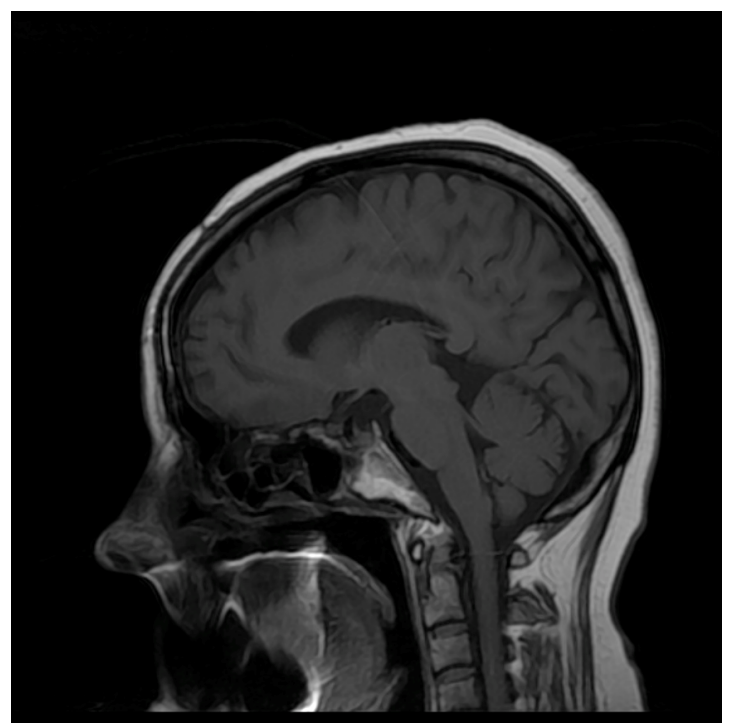

Figure 6. Total tumor removal with 2 months postop MRI scan

\section{Discussions}

Planum sphenoidale and tuberculum sellae meningiomas present a frequently encountered pathology of the anterior skull base, representing according to some authors 5 to $10 \%$ of all intracranial meningiomas $(1,2)$

These meningiomas give rise to an early visual pathology with relatively slow progression, but due to the fact that other symptoms are missing or are subtle they have a larger tendency to develop undiagnosed for longer periods of time (7, 9). In our study we have found that the headache is not a viable symptom, nor that its absence is a predictor for lesser tumoral size.

In our study we have included both tuberculum sellae and planum sphenoidale meningiomas as the anatomical landmarks are very close to each other, they displace the optic apparatus in similar fashion and were operated with the same microsurgical technique (unilateral subfrontal approach). 
DOI: 10.2478/v10282-012-0025-y

There are cases described in the literature of tuberculum sellae meningiomas misinterpreted as pituitary macroadenomas, but this was not the case in our study $(14,16)$.

All the cases in our study were operated via a fronto-pterional craniotomy positioned on the side with the most visual deficit, as other authors have mentioned $(15,16,17)$ we believe that this is the optimal surgical technique due to the fairly limited extendtof the craniotomy, familiarity of the neurosurgeon with the region, the early visualization of the tumor and the optic apparatus allowing for rapid identification of the important surgical landmarks which in turn resulted in good rates of tumor resection.

Out of the 18 cases operated $11(61,1 \%)$ cases presented improvement of the visual acuity whilst in $5(27,7 \%)$ cases the visual acuity remained stable, overall this results in a stabilization of the preoperative visual acuity in over $88 \%$ of the treated cases, a percentage that is in accordance with microsurgical series presented in the literature. $(6,16)$. All the patients in the early decompression group presented an increase in the visual acuity, whilst in the late decompression group the majority have presented stabilization of the visual deficit and only $22,25 \%$ presented improvement of the visual acuity. This is mostly because quickly diagnosed tumors are more likely to be of a lesser size and have not caused irreparable damage to the vascularization of the optic nerves.

Tumor removal was achieved in 77,7\% of cases, which is well between the described 56 to $100 \%$ margins found in the literature $(1,6,8)$. It should be mentioned that even though complete tumor removal was the proposed surgical target for all the surgical interventions in our study we feel that this should not be imperative and be preformed at the expense of higher morbidity.

Recurrence of the tumors was very low (1 case) and we believe this is due to the relative small amount of patients included in the study.

Advances in the field of endoscopy and the introductions of various extended transsphenoidal resection techniques have lately revolutionized the surgical management of the parasellar meningiomas $(3,5)$. We have no experience in our department with such approaches.

Indications for endoscopic approaches are tumors smaller than $2 \mathrm{~cm}$ situated on the midline with no extension into the optic canal and no vessels encased in tumor(5). Studies have shown that patients treated via extended endoscopic approaches might benefit from better rates of complete surgical resection and visual outcome whilst having the major drawback of having very high rates (29\%) of CSF fistulas (13).

For the time being we believe the role of microsurgical decompression is still the best choice of treatment providing optimal tumor management and visual recovery.
Corresponding author:
Dr. Adrian Florian Balasa
Head of Neurosurgery Departement, Targu Mures Clinical Emergency Hospital
Phone: +40265 212111 int. 297
+40740651053
Fax:+40265210621
E-mail:adrian.balasa@yahoo.fr
Address: Tg. Mures Clinical Emergency Hospital Neurosurgery Departement
Gheorghe Marinescu Street, no. 50, $3^{\text {rd }}$ floor. 


\section{References}

1. Al-Mefty O, Smith RR: Tuberculum sellae meningiomas, in Al-Mefty (ed): Meningiomas, New York, Raven Press, 1991, pp 395-411.

2. Al-Mefty O, Holoubi A, Rifai A, Fox JL: Microsurgical removal of suprasellar meningiomas. Neurosurgery 16:364-372, 1985.

3. Arai H, Sato K, Okuda, Miyajima M, Hishii M, Nakanishi H, Ishii H: Transcranial transsphenoidal approach for tuberculum sellae meningiomas. Acta Neurochir (Wien) 142:751-757, 2000.

4. Benjamin V, M.D. Russell M, M.D.: The microsurgical nuances of resecting tuberculum sellae meningiomas, Operative Neurosurgery, vol 56, 2005

5. Chi JH, McDermott MW. Tuberculum sellae meningiomas. Neurosurg Focus. 2003;14(6):e6.

6. de Divitiis, Enrico; Cavallo, Luigi M: Extended Endoscopic Transsphenoidal Approach for Tuberculum Sellae Meningiomas, Neurosurgery 62 (6): 1192-1201, 2008.

7. Fahlbusch R, Schott W: Pterional surgery of meningiomas of the tuberculum sellae and planum sphenoidale: surgical results with special consideration of ophthalmological and endocrinological outcomes. J Neurosurg. 2002 Feb;96(2):235-43.

8. Goel, Atul M.Ch: Tuberculum Sellae Meningioma: A Report on Management on the Basis of a Surgical Experience with 70 Patients. Neurosurgery 51: 13581364, 2002.

9. Jallo G I, MD, Benjamin V, M.D.: Tuberculum sellae meningiomas: microsurgical anatomy and aurgical technique, Neurosurgery vol 51, 2002.

10. Kitano M, Taneda M, Nakao Y. Postoperative improvement in visual function in patients with tuberculum sellae meningiomas: results of the extended transsphenoidal and transcranial approaches. J Neurosurg. 2007;107(2):337-346.
11. Kaye, AH, MD, PHD, Black, P., PHD Operative Neurosurgery, 1st edition, 1999.

12. Margalit NS, Lesser JB, Moche J, Sen C. Meningiomas involving the optic nerve: technical aspects and outcomes for a series of 50 patients. Neurosurgery. 2003;53(3):523-532.

13. Mathiesen T, Kihlstrom L. Visual outcome of tuberculum sellae meningiomas after extradural optic nerve decompression. Neurosurgery. 2006;59(3):570576.

14. Nasir Fatemi: Endonasal versus supraorbital keyhole removal of craniopharingiomas and tuberculum sellae meningiomas, Neurosurgery 2009 May;64(5 Suppl 2):269-84.

15. Pamir MN, Ozduman K, Belirgen M, Kilic T, Ozek MM. Outcome determinates of pterional surgery for tuberculum sellae meningiomas. Acta Neurochir (Wien). 2005;147(11):1121-1130.

16. Romani R, MD, PhD, Laakso A, MD, PhD Marko Kangasniemi M, MD, PhD, Niemela "M , MD, PhD, Hernesniemi J, MD, PhD: Lateral Supraorbital Approach Applied to Tuberculum Sellae Meningiomas: Experience With 52 Consecutive Patients, Neurosurgery 70, 2012.

17. Samii - Nakamura, Makoto M.D.; Roser, Florian M.D et al: Tuberculum Sellae Meningiomas: Clinical Outcome Considering Different Surgical Approaches, Neurosurgery 56(6): 1019-1029, 2006.

18. SchinK U Schick, W Hassler: Surgical management of tuberculum sellae meningiomas: involvement of the optic canal and visual outcome. J Neurol Neurosurg Psychiatry 2005;76:977-983.

19. Shunsuke T MD, Katsuyuki A, Hiroyuki K, Shigeru Y: Anterior Interhemispheric approach for Tuberculum Sellae Meningioma, Op Nerurosurgery 2011, 68.

20. Winn R., MD, Youmans Neurological Surgery 6th Edition, 2011, Elsevier. 